\title{
Optimization of the Active Control of Turboprop Cabin Noise
}

\author{
Jordi Romeu* \\ Universitat Politècnica de Catalunya, 08222 Terrassa, Spain \\ J. Ignacio Palacios $\ddagger$ \\ SENER, 08025 Barcelona, Spain \\ and \\ Andreu Balastegui $¥$ and Teresa Pamies \\ Universitat Politècnica de Catalunya, 08222 Terrassa, Spain
}

DOI: $10.2514 / 1 . C 032431$

\begin{abstract}
In this paper, a modified cost function is proposed in order to achieve the maximum noise attenuation using a set of secondary sources for a harmonically excited sound field. The modified cost function drives the error signal to the optimally attenuated sound field instead of minimizing the squared pressure. Moreover, changing the value to which the error signals must be driven allows change of the control strategy from global to local. The modified cost function requires the knowledge of the attenuated sound field, which is a condition that is well suited to narrowband noises, as is the case of turboprops. A numerical example of the application of the cost function is carried out using a finite element model/boundary element model of a real turboprop, with the goal of minimizing the interior sound field in the cabin to about $17 \mathrm{~m}^{3}$. A maximum averaged attenuation of $7 \mathrm{~dB}$ at blade-passage frequencies is achieved using six secondary sources and six error sensors, and $11 \mathrm{~dB}$ around the head of a seated crew member if the control system is tuned to achieve local control.
\end{abstract}

\section{Nomenclature}

$a_{p} \quad=$ primary source strength

$c_{0} \quad=$ speed of sound

$D_{n n} \quad=\quad$ damping factor of the $n$th mode

$E_{p} \quad=$ acoustic potential energy

$E_{p 0}=$ minimum potential energy achievable

$E_{p e 0}=$ minimum potential energy achievable in presence of errors

$E_{p p}=$ acoustic potential energy of the primary field

$E_{p s} \quad=$ acoustic potential energy of the controlled field

$H=$ Hermitian

$J_{p} \quad=$ cost function

$L^{p} \quad=\quad$ number of error sensors

$M \quad=$ number of secondary sources

$P \quad=$ acoustic pressure

$p_{J} \quad=$ acoustic pressure after minimizing the cost function

$p_{p} \quad=$ primary acoustic pressure

$p_{p}^{\prime}=$ error in the estimation of the primary acoustic pressure

$p_{s}=$ secondary acoustic pressure

$p_{V}=$ acoustic pressure inside the volume of the enclosure

$p_{\alpha 0}=$ acoustic pressure after minimizing the weighted potential energy

$p_{\Omega}=$ acoustic pressure inside the local volume

$p_{0} \quad=$ acoustic pressure after minimizing the potential energy

$q_{s}=$ secondary source strength

$q_{s J}=$ secondary source strength to minimize the cost function

$q_{s \Omega}=$ secondary source strength to minimize the local potential energy

$q_{s 0}=$ secondary source strength to minimize the potential energy
Received 15 May 2013; revision received 13 July 2015; accepted for publication 15 July 2015; published online XX epubMonth XXXX. Copyright $(\subset 2015$ by the American Institute of Aeronautics and Astronautics, Inc. All rights reserved. Copies of this paper may be made for personal or internal use, on condition that the copier pay the $\$ 10.00$ per-copy fee to the Copyright Clearance Center, Inc., 222 Rosewood Drive, Danvers, MA 01923; include the code 1533-3868/YY and \$10.00 in correspondence with the CCC.

*Associate Professor, Acoustic and Mechanical Engineering Laboratory, Mechanical Engineering Department.

${ }^{\dagger}$ Senior Researcher, Ingeniería y Sistemas; currently Hewlett Packard.

${ }^{\ddagger}$ Assistant Professor, Acoustic and Mechanical Engineering Laboratory, Mechanical Engineering Department. Colom 11.

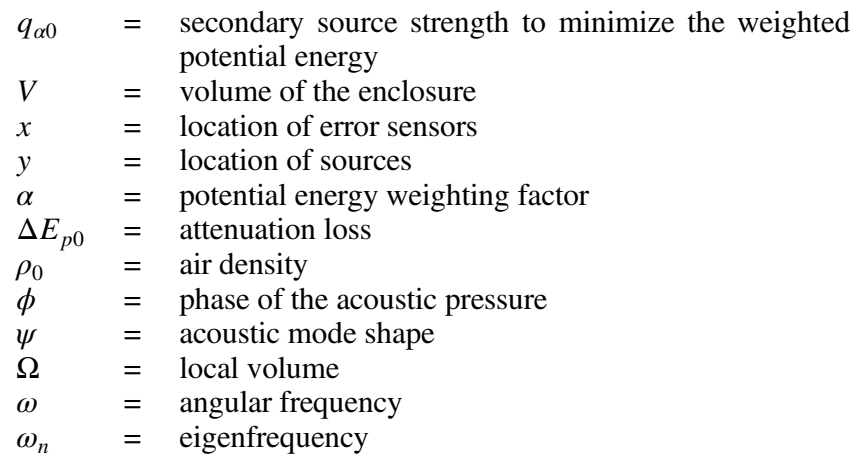

\section{Introduction}

$\mathbf{N}$ OISE levels in the passenger cabins of turbopropeller-driven aircraft are typically higher than the noise levels in comparable turbofan-powered aircrafts. This fact leads turboprop regional aircraft manufacturers to seek cabin noise reduction by passive and active means. Since the interior noise spectrum is dominated by tones occurring at multiples of the blade-passage frequency (BPF), the reduction of interior noise at these discrete frequencies can provide a significant improvement. Hence, the application of active techniques is very suitable in cabin noise control of turboprops and a good amount of research has been carried out in this field (see [1] for a review of the different active control strategies). Usually, a global reduction of the cabin noise is pursued and the best estimator of the efficiency of the active control system is the reduction of the acoustic potential energy. The efficiency of the active control system depends largely on the secondary actuators and error sensors configuration, and the optimization of the transducer configuration requires the knowledge of the cabin sound field.

The optimization of the number and location of secondary sources can be carried out by different strategies (see, for example, [2-4]), although it is usually performed by the minimization of a cost function $J_{p}$ defined by the summation of the squared pressure at a finite number of points, which could be the locations of the error sensors. When global control is pursued, the best results are usually obtained with acoustic error sensors, although other error sensors have been proposed [5].

However, there is no direct relation between the reduction of the squared pressure at a finite number of points (error sensors) and the reduction of potential energy $E_{p}$ [6]. Hence, the configuration of 
these sensors is crucial to guarantee that a reduction in the cost function comprises a reduction in the acoustic potential energy. Optimization procedures have been also applied to define the best configuration of error microphones. In some cases, the optimization routine is applied to define the optimal setup of the secondary sources and error microphones simultaneously, starting from a set of candidate positions for both kinds of transducers [6]. Other authors carry out the optimization independently: first, the optimum configuration of secondary sources is searched by minimizing the potential energy. Once it is defined, a procedure to optimize the error locations is applied in order to assure that a reduction in the cost function drives the reduction in the potential energy close to the optimum value defined by the secondary sources setup [7].

Regarding the optimization procedure, some extra rules have been pointed out by different authors, generally stating that the error sensors must be placed in the minimum of the optimally attenuated sound field [ $[\underline{8}, \underline{9}]$. However, Shafer et al. [10] verified that the achievement of null pressure in the case of real sound sources is not easy, and the performance of the active control system is consequently degraded. Finally, Martin and Gronier [11] established that it is possible to ensure that a reduction in $J_{p}$ leads to a reduction in $E_{p}$ by including a matrix that modifies the pressure at the error microphones. However, this matrix depends on the primary acoustic pressure at a very large number of points if $E_{p}$ must be attenuated in the whole enclosed volume.

However, all of these optimizations have been carried out in simple environments, such as rectangular enclosures [뭄, $\underline{11}]$ or free-field conditions [10]. The implementation of a complete set of transducers for actual turboprop cabin noise requires a large amount of experimental measurements [12-18] seeking for the best achievable configuration. Moreover, the number of error microphones needs to be greater than the number of secondary sources [19] in order to smooth the residual sound field and avoid local increases [20].

In the next section, a new cost function is proposed that ensures the maximum reduction of the acoustic potential energy that can be achieved by a set of secondary sources, using the same number of error sensors and secondary sources. The proposed control scheme requires the knowledge of the sound field. Therefore, it suits very well the case of stationary sound fields that typically takes place in turboprops cabin noise. Moreover, the cost function can be easily modified to prioritize the attenuation at some limited volumes of the cabin if desired. Once the cost function is derived, it is applied to the optimization of global active noise control of a turboprop cabin. Since the characteristics of the sound field are needed to carry out the optimization, a numerical model of turboprop cabin noise is briefly outlined.

\section{Active Noise Control Theory}

\section{A. Minimization of the Squared Pressure}

Typically, the active noise control system is aimed to minimize a set of error signals, which are proportional to the acoustic pressure, when microphones are used as error sensors. If $L$ error microphones are used, the vector of complex acoustic pressure at each sensor can be written as [21]

$$
\boldsymbol{p}=\boldsymbol{\psi}_{x}\left[\boldsymbol{a}_{p}+\boldsymbol{B} \boldsymbol{q}_{s}\right]
$$

where $\boldsymbol{p}^{T}=\left[p\left(\boldsymbol{x}_{1}\right), p\left(\boldsymbol{x}_{2}\right), \ldots p\left(\boldsymbol{x}_{L}\right)\right] ; \boldsymbol{\psi}_{x}$ is an $L \times N$ matrix for which the element $(1, n)$ is the value of the shape of the normal mode $n$ at the location of the $l$ error microphone; $\boldsymbol{a}_{p}$ is the vector of the $N$ primary complex mode amplitudes; $\boldsymbol{q}_{s}$ is the vector of the $M$ secondary sources strengths; and $\boldsymbol{B}$ is an $N \times M$ matrix that relates mode amplitudes with secondary point sources according to

$$
B_{n m}=\frac{\omega \rho_{0} c_{0}^{2}}{V\left[\omega c_{0} D_{n n}+j\left(\omega^{2}-\omega_{n}^{2}\right)\right]} \psi_{n}\left(\boldsymbol{y}_{m}\right)
$$

where $\psi_{n}\left(\boldsymbol{y}_{m}\right)$ is the value of the shape of the normal mode $n$ at the position of the secondary source $m, \omega$ is the angular frequency, $\omega_{n}$ and $D_{n n}$ are the eigenfrequency and the damping factor of the $n$th mode, $V$ is the volume of the cavity, $\rho_{0}$ is the air density, and $c_{0}$ is the speed of sound. The cost function that the controller tries to minimize is composed by the addition of squared pressure at these error microphones:

$$
\begin{aligned}
J_{p} & =\frac{V}{4 \rho_{0} c_{0}^{2} L}\left[\boldsymbol{q}_{s}^{H} \boldsymbol{B}^{H} \boldsymbol{\psi}_{x}^{H} \boldsymbol{\psi}_{x} \boldsymbol{B} \boldsymbol{q}_{s}+\boldsymbol{q}_{s}^{H} \boldsymbol{B}^{H} \boldsymbol{\psi}_{x}^{H} \boldsymbol{\psi}_{x} \boldsymbol{a}_{p}\right. \\
& \left.+\boldsymbol{a}_{p}^{H} \boldsymbol{\psi}_{x}^{H} \boldsymbol{\psi}_{x} \boldsymbol{B} \boldsymbol{q}_{s}+\boldsymbol{a}_{p}^{H} \boldsymbol{\psi}_{x}^{H} \boldsymbol{\psi}_{x} \boldsymbol{a}_{p}\right]
\end{aligned}
$$

where $H$ means Hermitian. Following the development of Nelson et al. [22], the set of secondary source strengths that minimize this cost function is

$$
\boldsymbol{q}_{s J}=-\left[\boldsymbol{B}^{H} \boldsymbol{\psi}_{x}^{H} \boldsymbol{\psi}_{x} \boldsymbol{B}\right]^{-1} \boldsymbol{B}^{H} \boldsymbol{\psi}_{x}^{H} \boldsymbol{\psi}_{x} \boldsymbol{a}_{p}
$$

\section{B. Minimization of Potential Energy}

For the steady state, the potential energy of an enclosed sound field excited by the primary sources $\boldsymbol{a}_{p}$ and a set of secondary point sources $\boldsymbol{q}_{s}$ can be written in matrix form as [21]

$$
E_{p}=\frac{V}{4 \rho_{0} c_{0}^{2}}\left[\boldsymbol{q}_{s}^{H} \boldsymbol{B}^{H} \boldsymbol{B} \boldsymbol{q}_{s}+\boldsymbol{q}_{s}^{H} \boldsymbol{B}^{H} \boldsymbol{a}_{p}+\boldsymbol{a}_{p} \boldsymbol{B} \boldsymbol{q}_{s}+\boldsymbol{a}_{p}^{H} \boldsymbol{a}_{p}\right]
$$

Assuming the general condition that $N>M$, the optimal set of secondary source strengths that minimize $E_{p}$ is given by

$$
\boldsymbol{q}_{s 0}=-\left[\boldsymbol{B}^{H} \boldsymbol{B}\right]^{-1} \boldsymbol{B}^{H} \boldsymbol{a}_{p}
$$

It is clear that the set of secondary source strengths that minimizes the cost function is not the same as the one that minimizes the potential energy.

\section{Minimization of Potential Energy Through a Limited Number Of Error Sensors}

The residual pressure at the error sensors after its minimization according to Eq. (4), $\boldsymbol{p}_{J}$, is uniquely defined as

$$
\boldsymbol{p}_{J}=\boldsymbol{\psi}_{x}\left[\boldsymbol{a}_{p}+\boldsymbol{B} \boldsymbol{q}_{S J}\right]
$$

On the other hand, the vector of sound pressures at the error sensors when the potential energy of the enclosure is minimized, following Eq. (ㅁ), is uniquely

$$
\boldsymbol{p}_{0}=\boldsymbol{\psi}_{x}\left[\boldsymbol{a}_{p}+\boldsymbol{B} \boldsymbol{q}_{s 0}\right]
$$

Since the relation between the pressure at any point and the strength of the secondary source is uniquely defined, it is clear that if the other residual value of $\boldsymbol{p}$ different than $\boldsymbol{p}_{0}$ is reached, the attenuated sound field is not the optimally attenuated one. Thus, in order to ensure that the optimal attenuation is reached, the cost function must force the residual vector $\boldsymbol{p}_{J}$ to be $\boldsymbol{p}_{0}$, and this statement is equivalent to minimize the following corrected cost function:

$$
J_{p}=\frac{V}{4 \rho_{0} c_{0}^{2} L} \sum_{l=1}^{L}\left|p\left(x_{l}\right)-p_{0}\left(x_{l}\right)\right|^{2}
$$

This means that the control system should be arranged to not minimize the summation of the squared pressures of the error sensors but to drive these pressures to the values given by the optimally attenuated sound field. If Eq. (9) is written in matrix form,

$$
J_{p}=\frac{V}{4 \rho_{0} c_{0}^{2} L} \sum_{i=1}^{L}\left[\left(\boldsymbol{p}_{p}+\boldsymbol{p}_{s}\right)-\boldsymbol{p}_{0}\right]_{i}^{2}=\left[\boldsymbol{q}_{s r}^{H} \boldsymbol{B}^{H} \boldsymbol{\psi}_{x}^{H} \boldsymbol{\psi}_{x} \boldsymbol{B} \boldsymbol{q}_{s r}\right]
$$

where $\boldsymbol{p}_{p}$ and $\boldsymbol{p}_{s}$ are the pressure vectors at the error sensors due to primary and secondary sources, respectively; and $\boldsymbol{q}_{s r}=\boldsymbol{q}_{s}-\boldsymbol{q}_{s 0}$. It is easily seen that the minimum value of the cost function is zero and it is 
found when $\boldsymbol{q}_{s r}=\boldsymbol{q}_{s}-\boldsymbol{q}_{s 0}=0$, which leads to the solution of $\boldsymbol{q}_{s}=\boldsymbol{q}_{s 0}$. Thus, the optimal global attenuation remains guaranteed for any value of $L$ (being $L \geq M$ ) and for any location of the error sensors, since the values of $\boldsymbol{B}$ and $\boldsymbol{\psi}_{x}$ do not have to fulfill any specific condition. Note that the previous researchers $[\underline{8}-10,23]$, who identified the null pressure points of the optimally attenuated sound field as the best location for the error sensors, found the particular case of Eq. (10) in which $\boldsymbol{p}_{0}$ was equal to zero. Thus, the cost function represented by Eq. (10) is a generalized approach of this particular procedure and gives the chance of placing the error sensors in points of relatively high sound pressure of the optimally attenuated sound field, thus avoiding the limiting effect of the broadband noise floor on the efficiency of the system [23].

\section{Adaptive Volume Control}

The performance of active noise control systems in large rooms tends to be reduced or is even unfeasible above a certain frequency. In that situation, local control becomes the alternative to ensure attenuation inside a finite volume of a room, without increasing the acoustic potential energy of the rest of the room. This strategy requires the secondary source to be placed close to the error sensors [21]; otherwise, an increase of sound pressure can occur out of the local zone of control [24]. This requirement is a severe limitation on the applicability of local control, and a lot of experimental work has to be carried out to find positions of error sensors that ensure attenuation in areas: for example, comprising the passenger heads $[12,13]$. The modification in the cost function defined by Eq. (10) can transform one active control system designed for global control into one focused on local control. This would have the advantage of having the secondary sources and error sensors far from the zone of control or even let an active system switch from a global to a local control strategy.

Let a control volume $\Omega$ be defined inside a room volume $V$ with boundary $S$. The primary sound field is continuously defined inside $V$; that is, there is a continuity in both pressure $P_{\sigma}^{V}=P_{\sigma}^{\Omega}$ and particle velocity $u_{\sigma}^{V}=u_{\sigma}^{\Omega}$, where $\sigma$ is the boundary of $\Omega$. The secondary sources and error sensors are placed on the boundary $S$ of the volume $V$ (Fig. 1). The goal of the active control system is to reduce the potential energy inside the volume $\Omega$, approximated in this case by the cost function

$$
J_{p}=\frac{V}{4 \rho_{0} c_{0}^{2} L_{\infty}} \boldsymbol{p}_{\Omega}^{H} \boldsymbol{p}_{\Omega}
$$

where the vector $\boldsymbol{p}_{\Omega}$ is defined by the acoustic pressure calculated at a very large number $L_{\infty}$ of calculation points inside the control volume in order to obtain a correct estimation of the acoustic potential energy. The potential energy of the control zone cannot be written as a function of the modal amplitudes as in Eq. (5), since only one part of the spatial modal function participates in the potential energy of the (unbounded) control volume. The sound pressure inside the control zone can be written as

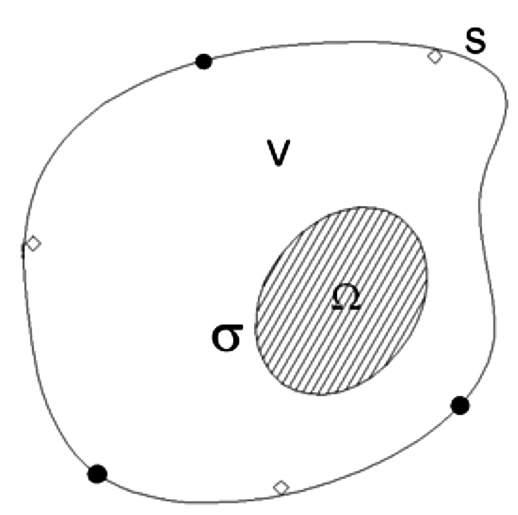

Fig. 1 Local volume of attenuation $\Omega$ inside an enclosure $V$ with secondary sources (black dots) and error sensors placed in the enclosure boundary.

$$
\boldsymbol{p}=\boldsymbol{\psi}_{\Omega x}\left[\boldsymbol{a}_{p}+\boldsymbol{B} \boldsymbol{q}_{s}\right]
$$

And, the set of secondary source strengths that minimizes the cost function is

$$
\boldsymbol{q}_{\Omega s}=-\left[\boldsymbol{B}^{H} \boldsymbol{\psi}_{\Omega x}^{H} \boldsymbol{\psi}_{\Omega x} \boldsymbol{B}\right]^{-1} \boldsymbol{B}^{H} \boldsymbol{\psi}_{\Omega x}^{H} \boldsymbol{\psi}_{\Omega x} \boldsymbol{a}_{p}
$$

This set of strengths is obviously different from $\boldsymbol{q}_{s 0}$ in Eq. (6). This result can also lead to an increase of the sound pressure outside the zone of control. To avoid this unwanted situation, the target of the active control is modified to minimize a weighted function of the cost function including measurement points inside and outside the zone of control, as

$$
J_{p}=\frac{V}{4 \rho_{0} c_{0}^{2}}\left[\alpha \frac{\boldsymbol{p}_{\Omega}^{H} \boldsymbol{p}_{\Omega}}{L_{\infty}}+(1-\alpha) \frac{\boldsymbol{p}_{V}^{H} \boldsymbol{p}_{V}}{R_{\infty}}\right]
$$

where $\boldsymbol{p}_{V}$ is the vector of acoustic pressure calculated at a large number $R_{\infty}$ of calculation points outside $\Omega$, and $\alpha$ is the weighting value that gives more or less importance to the contribution of the two volumes and should avoid increases of potential energy in both volumes if it is properly selected. The value of $\alpha$ should be calculated in order to obtain the maximum attenuation of the potential energy inside the volume $\Omega$ without increasing the potential energy outside that volume. For that value of $\alpha$, the set of secondary strengths that ensures the maximum attenuation (given a set of secondary source strengths) inside the control volume is $\boldsymbol{q}_{\alpha 0}$, and the minimized residual field is defined for that set of secondary strengths. For a practical control system, the cost function must be defined by a limited number of measurement points (error sensors) and, following the same argument that lead to Eq. (10), the optimal set of secondary strengths is only obtained if the cost function is adapted to drive the pressures at the error sensors to the values corresponding to the optimally attenuated sound field given by the set $\boldsymbol{q}_{\alpha 0}$ Therefore, the cost function should be

$$
J_{p}=\frac{V}{4 \rho_{0} c_{0}^{2} L} \sum_{l=1}^{L}\left|p\left(x_{l}\right)-p_{\alpha 0}\left(x_{l}\right)\right|^{2}
$$

where $p_{\alpha 0}\left(x_{i}\right)$ is the residual pressure of the optimally attenuated sound field given by the set $\boldsymbol{q}_{\alpha 0}$. Note that neither the secondary sources nor the error sensors must be placed inside the control volume $\Omega$.

The cost function expressed by Eqs. (10) or (15) is really the same and depends only on the residual pressure desired at each error sensor. In fact, a controller based in such a function can change from global control mode to local control mode, changing the target values from $p_{0}\left(x_{i}\right)$ to $p_{\alpha 0}\left(x_{i}\right)$. These values must be previously known; therefore, the practicality of the method is limited to the situations in which the response of the enclosure is known. These quantities can be determined by using numerical methods such as the finite element method or the boundary element method, especially in the low-frequency range. All these limitations are well suited for the control of stable sound fields, i.e., narrowband disturbances such as turboprop cabin noise. Note that, if the disturbance is perfectly periodic and the plant response does not change, the optimal control can be achieved with a fixed controller, and the adaptive controller would be unnecessary. However, in real applications, an adaptive system must be used because real signals change slowly [25] and the response of the electroacoustic components can vary due to physical changes [26,27].

\section{Numerical Model}

The optimization procedure is applied to a numerical model of a turboprop military transport aircraft conceived for a tactical airlift capable of operating on short and semiprepared runways. Typical missions include the transport of troops and cargo, medical evacuation, humanitarian missions, and maritime patrol. Its main features are listed in Table $\underline{1}$. The nominal propeller rotational speed is $120 \mathrm{~Hz}$ 
Table 1 Turboprop main features

\begin{tabular}{lc}
\hline \hline Parameter & Value \\
\hline Maximum takeoff weight & $23,200 \mathrm{~kg}$ \\
Maximum payload & $9250 \mathrm{~kg}$ \\
Powerplant & $2 \times \mathrm{PW} 127 \mathrm{G}$ \\
Maximum cont. power & $2645 \mathrm{SHP}$ \\
Propeller speed & $1200 \mathrm{rpm}$ \\
Propeller blades & 6 \\
Propeller diameter & $3932 \mathrm{~mm}$ \\
Maximum cruise speed & $480 \mathrm{~km} / \mathrm{h}$ \\
Length & $24.5 \mathrm{~m}$ \\
Span & $25.8 \mathrm{~m}$ \\
Height & $8.66 \mathrm{~m}$ \\
\hline \hline
\end{tabular}

that, combined with high power and reduced clearance between the propeller and the fuselage, causes cabin A-weighted levels to be driven by BPFx1 and BPFx2, at least for high-power flight conditions where interior noise is maximum. The room in which a reduction of noise is desired (Fig. 2) is approximately centered at the propeller plane; its approximate size is $3 \mathrm{~m}$ long, $2.7 \mathrm{~m}$ wide (maximum), and $1.9 \mathrm{~m}$ high (maximum), and it is devoted to control tasks carried out by one crew member.

A finite element model (FEM) of the whole fuselage structure and a representation of the interior and exterior acoustic domains using boundary elements [boundary element method (BEM)] is available for that model. Detailed information of the model and its experimental validation can be found in [28], although it will be outlined in this section. The aircraft structural FEM contained a fine mesh for a fuselage section comprising 10 frames that included the propeller plane. The element size of this mesh was dimensioned for reproducing structural dynamic behavior up to $400 \mathrm{~Hz}$ (approximately $25 \mathrm{~mm}$ ). A coarser mesh based on an existing dynamic model [29] was used for the rest of the aircraft for the purpose of improving boundary conditions at the extremes of the section of interest. The complete FEM contained approximately 200,000 elements and $800,000 \mathrm{deg}$ of freedom. A snapshot of the complete FEM of the aircraft is given in Fig. 2. The number of normal modes obtained with the complete aircraft structural FEM reached approximately 2000 modes up to $250 \mathrm{~Hz}$ ( 36 acoustic modes up to $150 \mathrm{~Hz}$ ). The boundary element representation used for the coupled FEM/BEM was created based on the internal cabin geometry of the aircraft used for testing. System consoles and equipment racks were included in the model. Subdomains were created based on the geometrical position of the floor and roof panels.

The coupled vibroacoustic simulations were all performed using LMS Virtual Lab "Acoustic Harmonic Toolbox" pre- and postprocessing capabilities. Modal analysis of FEMs was performed using MSC NASTRAN. The frequency range of interest for the coupled simulation was set up to $150 \mathrm{~Hz}$, whereas the structural normal modes of the aircraft were calculated up to $250 \mathrm{~Hz}$. The numerical model

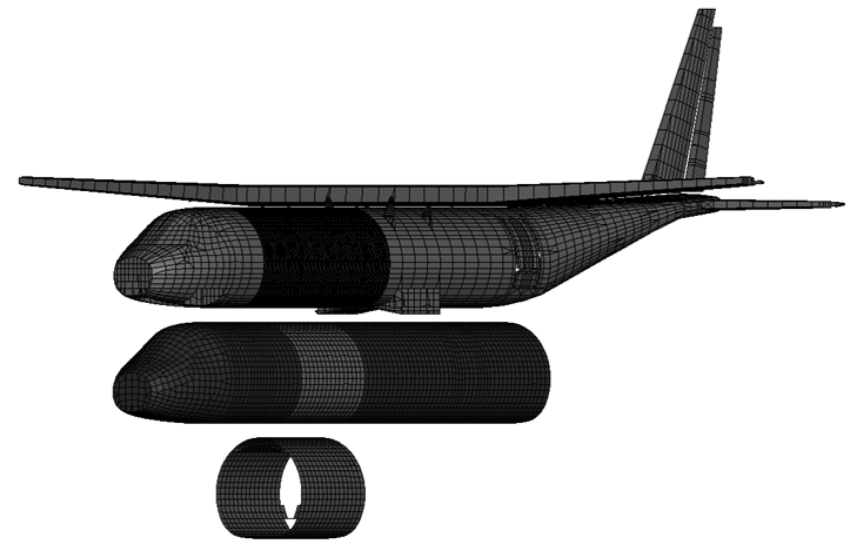

Fig. 2 Control region (below) and representation of the different meshes used in LMS Virtual Lab and LMS Sysnoise for coupled FEM/ BEM simulations.
Table 2 FEM/BEM input data

\begin{tabular}{lc}
\hline \hline Parameter & Value \\
\hline Temperature & $20^{\circ} \mathrm{C}$ \\
Atmosphere pressure & $843,036 \mathrm{~Pa}$ \\
Air density & $1 \mathrm{~kg} / \mathrm{m}^{3}$ \\
Sound speed in air & $343.3 \mathrm{~m} / \mathrm{s}$ \\
\hline \hline
\end{tabular}

(some input data can be seen in Table 2) was validated through a ground test using controllable synthetic sources (e.g., electrodynamic shakers and loudspeakers). For this study, however, the eventual acoustic loads induced by the synchronized propellers (phase shift of $180 \mathrm{deg}$ ) are introduced in the aircraft FEM/BEM by means of a deterministic point source [30] of arbitrary amplitude; therefore, the absolute values shown in the following sections are also arbitrary

Figure 3 shows the acoustic field (of arbitrary amplitude) outside the cabin in the blade plane at the BPF; and Fig. 4 shows the sound field inside the cabin (of arbitrary amplitude), at $\overline{\mathrm{BPF}}$, and at a height of $1.2 \mathrm{~m}$ above the cabin floor, corresponding to the height of the seated crew member. It is seen that the interior sound field is not smooth, suggesting a low modal sound field. The reverberation time of the cabin was experimentally measured giving a Schroeder's frequency value of $230 \mathrm{~Hz}$ [31]. This result confirms that the cabin sound field fulfills the low modal condition and that global control is achievable inside the cabin [32]. Finally, the comparison between measured and calculated cabin noise levels gives an averaged error of about $5 \%$ in the estimation of the acoustic pressure (linear scale) with a standard deviation of about $11 \%$ (linear scale).

\section{Method}

\section{A. Optimization of the Secondary Sources}

The potential energy of the cavity is estimated through Eq. (11) (extended to the volume $V$ of the cavity) by using 3009 measurement points from the BEM. The optimal secondary strengths are calculated through Eq. (13), and the attenuation $R$ is defined by the reduction of potential energy so that

$$
R=10 \log \left(\frac{E_{p p}}{E_{p s}}\right)
$$

where $E_{p p}$ is the acoustic potential energy of the primary field, and $E_{p s}$ is the acoustic potential energy of the optimally attenuated sound field.

The candidate locations for the secondary acoustic sources are selected according to the surface free of equipment in the ceiling and wall panels, in addition to considering a minimum distance between two correlative positions of $0.8 \mathrm{~m}$. Optimal attenuations are

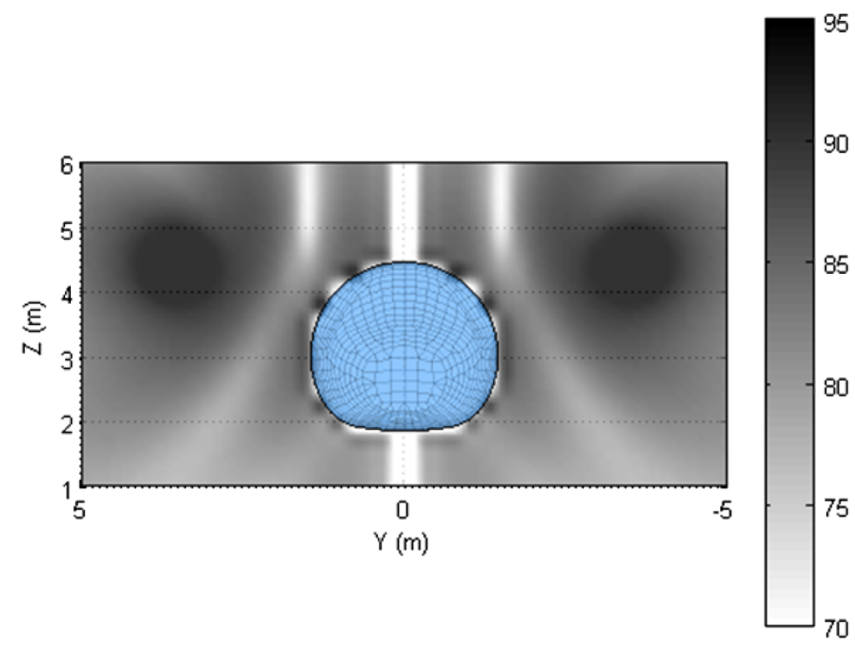

Fig. 3 Exterior noise field (BPF) at the blade plane (in decibels, with arbitrary amplitude). 


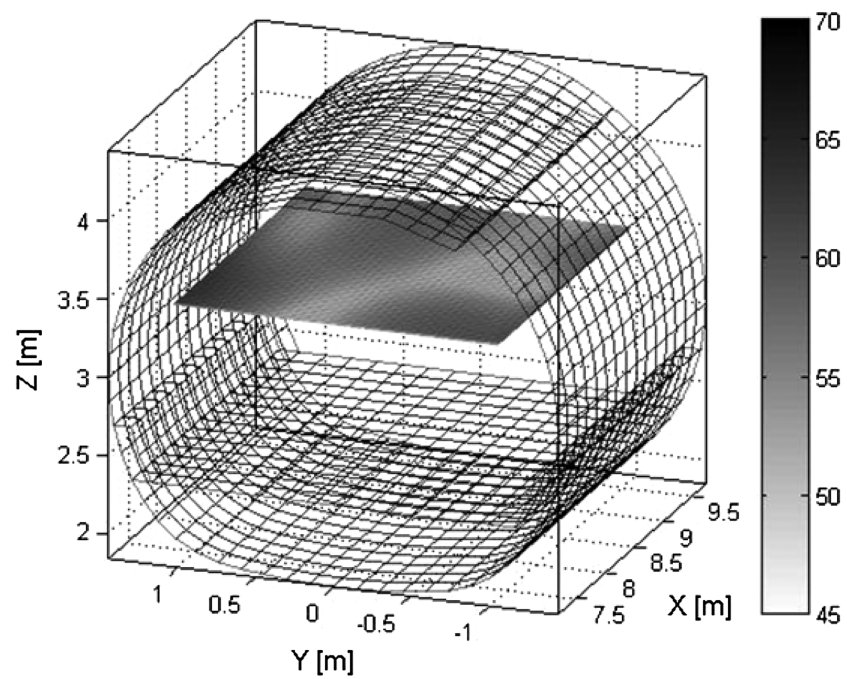

Fig. 4 Primary acoustic field (BPF) at $1.2 \mathrm{~m}$ above the cabin floor (in decibels, with arbitrary amplitude).

calculated for all combinations of loudspeaker positions such that the total number of secondary sources is varied from 1 to a maximum of 21 locations. However, calculations are truncated at $M$ secondary sources when the increase of attenuation is lesser than $0.5 \mathrm{~dB}$ compared to the best configuration of $M-1$ secondary sources.

\section{B. Robustness}

Once the secondary sources are located in their optimal positions, the next step is to place the error sensors. According to Eq. (9), there is no restriction in the location of error microphones, and its number can be equal to the number of secondary sources. However, this statement holds if the control system is designed for a uniquely defined working point, which can be called the reference condition [10] but, in real application, this reference condition is not completely defined without considering the uncertainty of the plant, that can reduce the efficiency of the control system [32]. It is possible that the location of the error sensors plays some role in the robustness of the control system. To investigate the effect of the unstructured errors [33], calculations are conducted in which the model is formed by adding a vector of Gaussian random numbers to the reference primary field. Thus, a vector error $\boldsymbol{p}_{p}^{\prime}$ is added to $\boldsymbol{p}_{p}$ in order to obtain a deviation of the reference situation of the primary acoustic field. Specifically, the elements $\hat{c}_{i j}$ of the vector error $\boldsymbol{p}_{p}^{\prime}$ are randomly calculated from a normal distribution defined by $N\left(0.05 c_{i j}, 0.11 c_{i j}\right)$, where $c_{i j}$ is the (complex) element of $\boldsymbol{p}_{p}$. These values approximate the measured error between the numerical model and the experimental values. Note that, under these circumstances, the cost function is written as

$$
J_{p}=\frac{V}{4 \rho_{0} c_{0}^{2} L} \sum_{i=1}^{L}\left[\left(\boldsymbol{p}_{p}+\boldsymbol{p}_{p}^{\prime}+\boldsymbol{p}_{s}\right)-\boldsymbol{p}_{0}\right]_{i}^{2}
$$

As a result, the set of secondary strengths that minimizes the cost function is no longer $\boldsymbol{q}_{s 0}$ and the performance of the optimally designed control system is reduced.

Table 3 Primary $p$ (arbitrary amplitude) and optimally attenuated acoustic field pressures $p_{0}$, and their phases ( $\phi$ and $\left.\phi_{0}\right)$, at the error sensors and optimal secondary sources strengths and their phases $\phi_{0^{\mathrm{a}}}$

\begin{tabular}{lccccccc}
\hline \hline Error & $p, \mathrm{~dB}$ & $\phi, \mathrm{rad}$ & $p_{0}, \mathrm{~dB}$ & $\phi_{0}, \mathrm{rad}$ & Source & $q_{s 0}, \mathrm{~m}^{3} / \mathrm{s}$ & $\phi_{q}, \mathrm{rad}$ \\
\hline 1 & 54.1 & 0.47 & 62.3 & -1.25 & 1 & 0.0068 & 0.44 \\
2 & 59.6 & -0.40 & 60.0 & -1.32 & 2 & 0.0045 & 0.53 \\
3 & 47.7 & 1.26 & 49.0 & -0.36 & 3 & 0.0013 & -0.49 \\
4 & 62.9 & 1.41 & 58.6 & 1.25 & 4 & 0.0046 & 0.42 \\
5 & 57.8 & -0.73 & 53.5 & 1.03 & 5 & 0.0027 & 0.66 \\
6 & 55.3 & -0.18 & 55.5 & 0.45 & 6 & 0.0018 & 1.30 \\
\hline \hline
\end{tabular}

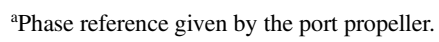

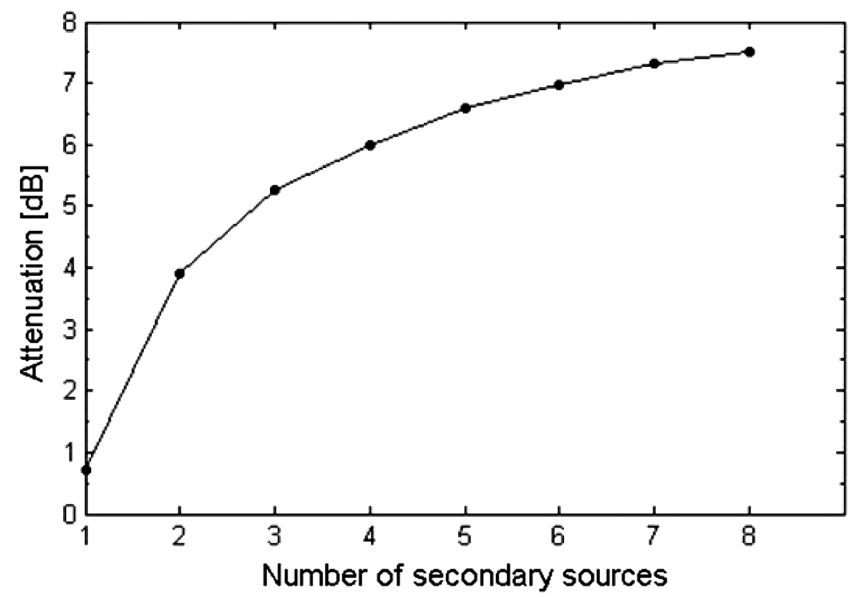

Fig. 5 Reduction of the acoustic potential energy vs number of secondary sources.

The robustness of the system can be numerically defined as the difference between the maximum possible attenuation given a set of secondary sources and the eventual attenuation obtained if the primary field is not the one considered as the reference condition. This difference can be expressed as the relation between the acoustic potential energy of the residual acoustic field when the error is induced in the reference primary field $E_{p e 0}$ and the acoustic potential energy of the optimally reduced sound field $E_{p 0}$. In this text, this relation is expressed in decibel scale and defined as the attenuation loss $\Delta E_{p 0}$, so that $\Delta E_{p 0}=10 \log \left(E_{p e 0} / E_{p 0}\right)$. Thus, positive values of $\Delta E_{p 0}$ mean a reduction of the attenuation (a degradation of the efficiency); and the greater the attenuation loss, the lesser the robustness.

Therefore, once the secondary sources are optimized, the location of the error sensors is defined by the set of error sensors that give the most robust control system design from a 100 different combinations of error sensors. Each defined set of error sensors accomplishes two different conditions: the distance between the microphones is at least $0.8 \mathrm{~m}$, and they are placed in the ceiling or wall plates (in order to not invade the room space). The robustness of each set of error sensors is determined by calculating its averaged attenuation loss for 100 different primary fields defined by Eq. (17).

\section{Results}

The maximum attenuation achievable as a function of the number of secondary sources can be seen in Fig. 5. Calculations are truncated at eight secondary sources because the increase of attenuation using such a number of secondary sources with respect to the maximum attenuation using seven secondary sources is only $0.2 \mathrm{~dB}$. The maximum achievable attenuation for eight secondary sources is $7.5 \mathrm{~dB}$; meanwhile, for seven and six secondary sources, the maximum attenuations are 7.3 and $7.0 \mathrm{~dB}$, respectively. Considering the averaged error committed in calculating the primary field, it seems that an increase of attenuation of about $0.5 \mathrm{~dB}$ is not worth the effort, and a reasonable number of six secondary sources is chosen as a reference condition. Specifically, the location of the six secondary sources is shown in Fig. 6 and the optimally attenuated sound field at $1.2 \mathrm{~m}$ height is shown in Fig. 7. The controlled acoustic field is smoother than the primary field, and the potential energy reduction is achieved by the reduction of the zones of maximum noise level of the primary field. This suggests a reduction of the dominant contributing modes by the action of the secondary sources. Table 3 summarizes the initial pressures and the optimally attenuated pressures at the error sensors (used to define the cost function), as well as the secondary sources strengths, which ensures the minimization of the primary acoustic field. It must be noted that some increases of the pressure at the error microphones after the optimal control must be accepted in order to achieve the best global control.

Since the six error sensors can be located elsewhere from the point of view of performance, the optimization of its configuration (number 


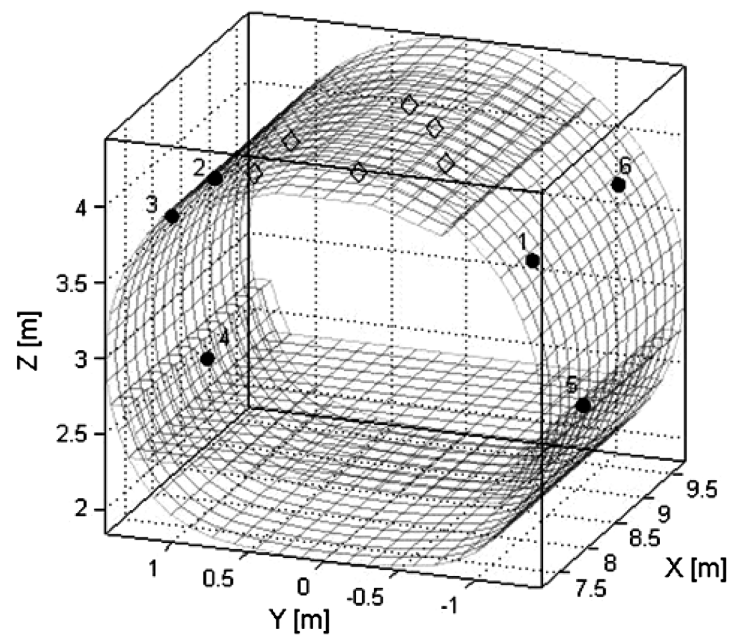

Fig. 6 Location of the secondary sources (black dots) and error sensors (void rhombus).

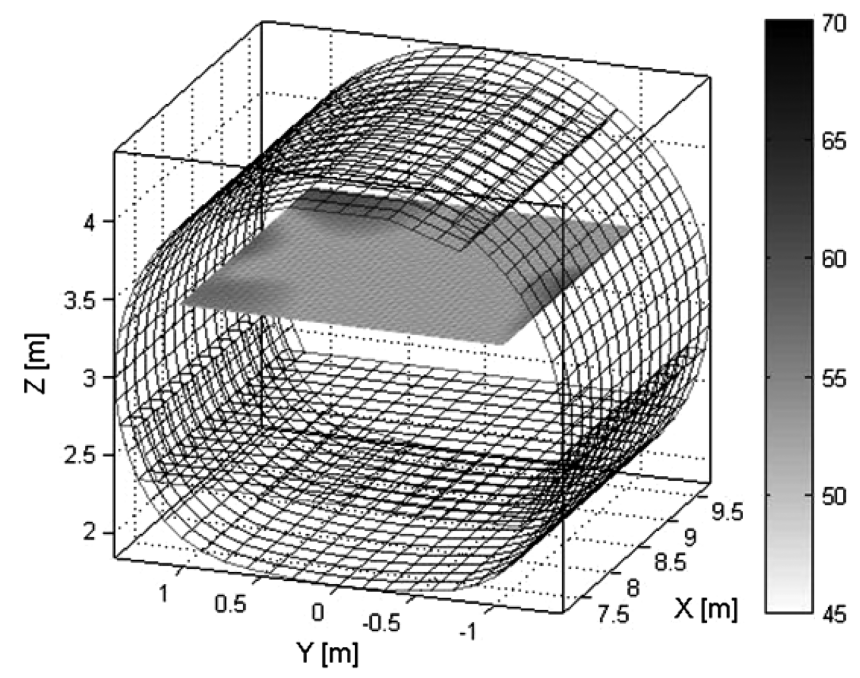

Fig. 7 Optimally attenuated acoustic field at the plane defined by $z=1.2 \mathrm{~m}$ (in decibels).

and location) is carried out as a function of the robustness of the control system. As a starting point, the best configuration of the six secondary sources is used, and their strength is now adjusted to minimize the cost function defined by Eq. (17) for the 100 different sets of six error sensors. The effect on the robustness of the deviation in the reference primary acoustic field gives an averaged attenuation loss of $0.1 \mathrm{~dB}$. The best configuration gives an averaged (over the 100 different randomly modified primary fields) attenuation loss of $0.002 \mathrm{~dB}$, and the worst one gives an attenuation loss of $0.8 \mathrm{~dB}$. Considering the small values of attenuation loss induced by the error between real and calculated primary fields, it seems that the location of the error sensors has little effect on the robustness of the system.

If desired, local control can be applied to around the crew member head (Fig. 8) without increasing the sound pressure in the rest of the

Table 4 Primary $\boldsymbol{p}$ and optimally attenuated sound field pressures $p_{0}$ at the error sensors and optimal secondary source strengths for a locally optimized active control with four secondary sources ${ }^{a}$

\begin{tabular}{lccccccc}
\hline \hline Error & $p, \mathrm{~dB}$ & $\phi, \mathrm{rad}$ & $p_{0}, \mathrm{~dB}$ & $\phi_{0}, \mathrm{rad}$ & Source & $q_{s 0}, \mathrm{~m}^{3} / \mathrm{s}$ & $\phi_{q}, \mathrm{rad}$ \\
\hline 1 & 54.1 & 0.47 & 60.3 & 1.03 & 1 & 0.0068 & 0.44 \\
2 & 59.6 & -0.40 & 47.3 & -1.44 & 2 & 0.0045 & 0.53 \\
3 & 47.7 & 1.26 & 65.6 & 1.25 & 3 & 0.0065 & -1.39 \\
4 & 62.9 & 1.41 & 55.4 & -0.88 & 4 & 0.0046 & 0.42 \\
\hline \hline
\end{tabular}

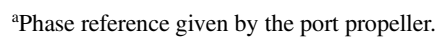

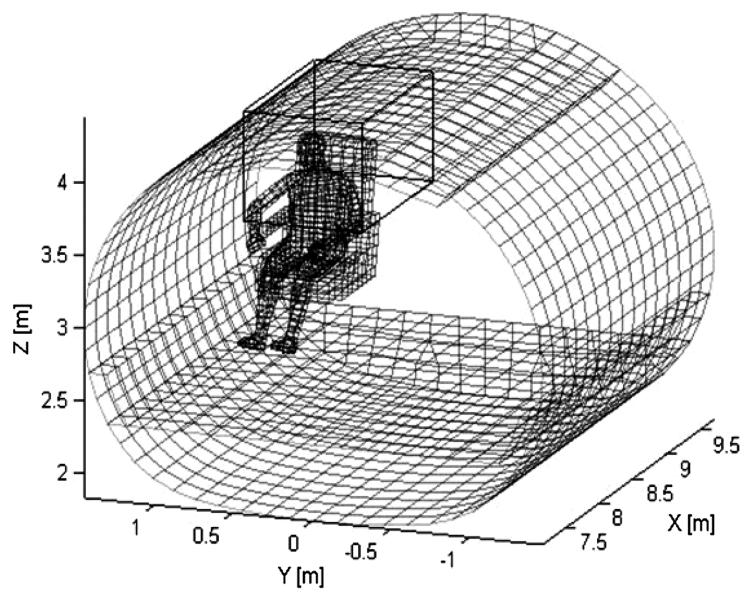

Fig. 8 Selected region for local control. The model of the human body is not included in the calculations.

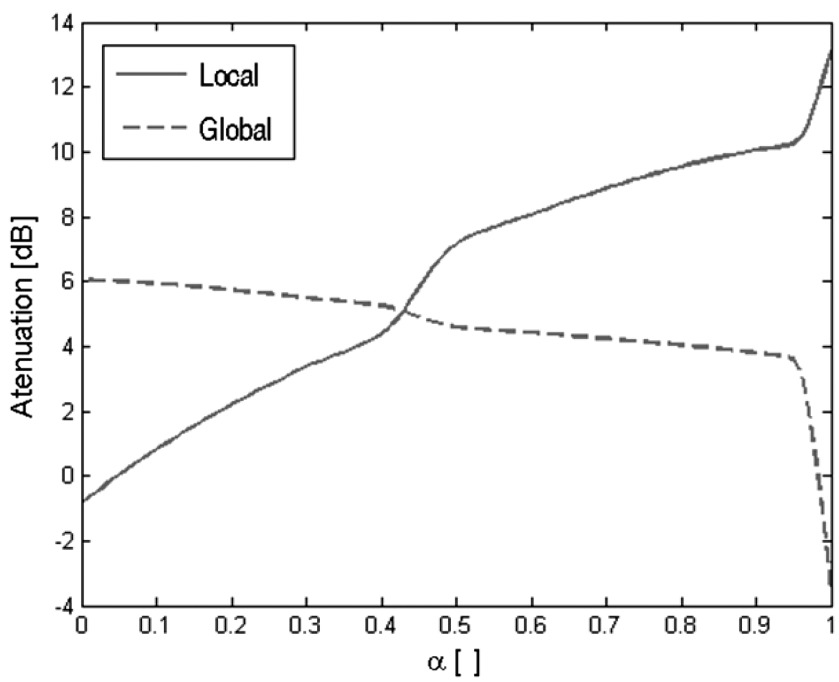

Fig. 9 Reduction of potential energy in the region around the crew member head (local) and in the rest of the room (global) vs the weighting factor $\alpha$.

volume. Since the control volume becomes smaller when switching from global control to local control, a higher attenuation is expected inside the volume of local control compared to the attenuation achieved when global control is pursued. The first step is to calculate the value of the weighting factor $\alpha$ of Eq. (14), which reduces the potential energy in the area surrounding the worker without increasing the acoustic potential energy in the rest of the room. Using, in that case, the configuration of the best four secondary sources, the cost function defined by Eq. (14) is minimized for different values of $\alpha$. The acoustic potential energy at the volume $\Omega$ (surrounding the crew member) and at the rest of the room $V$ are estimated through the pressure calculated at 240 and 2769 points, respectively, which is a squared volume of a side of $0.6 \mathrm{~m}$. Results are plotted in Fig. 9, and it is seen that, if the local control is defined without considering the acoustic potential energy at the rest of the room $(\alpha=1)$, high local attenuation is achieved, but at a cost of increasing the potential energy of the rest of the room up to $4 \mathrm{~dB}$. If the local control is prioritized, a value of $\alpha=0.95$ gives an attenuation around $10 \mathrm{~dB}$ in the local region without increasing the potential energy at the rest of the room. For a given $\alpha$, there is a set of optimal secondary strengths that minimizes the cost function of Eq. (14), and thus gives new residual pressures at the error sensors, which are the values to be used in the cost function of the active control system defined by Eq. (17). Table 4 shows the new values (for $\alpha=0.95$ ) of the residual pressures at the error sensors and the optimal secondary strengths that ensure a maximization of the local attenuation around the worker's head without 


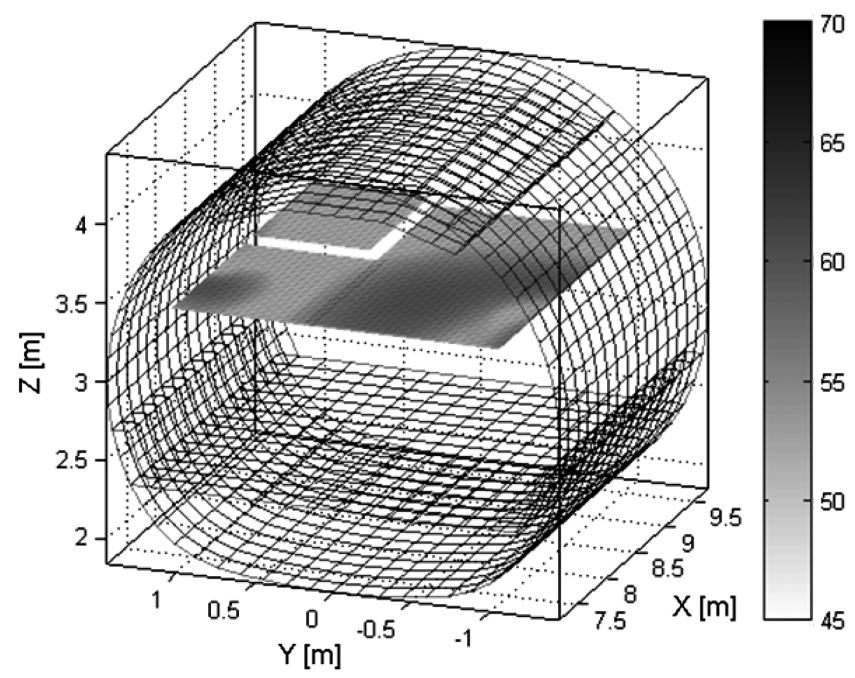

Fig. 10 Optimally attenuated local sound field at the plane defined by $z=1.2 \mathrm{~m}$.

increasing the potential energy in the rest of the cabin, without changing the location of the secondary sources and error sensors. Note that only four (whatever they are) of the six error sensors are needed. Figure 10 also shows the residual sound fields after local attenuation. The controlled acoustic field is uniform in the region close to the head, and it can be seen that the residual field outside the local region of attenuation suffers some modification, but noise levels are similar to those of the primary field (Fig. 4). Thus, the active control system rearranges the contributing modes in order to avoid the presence of zones of high noise level inside the local region of control (as it does when global control is attempted) but also avoiding the increase outside this local region.

\section{Conclusions}

A modified cost function for the global active control of enclosed sound fields is proposed, which ensures achievement of the optimal attenuation that can be obtained by a set of secondary sources. The usual cost function based on the summation of squared pressures given by error microphones is modified by subtracting at each pressure the value of the pressure corresponding to the optimally attenuated sound field, which depends only on the number and location of the secondary sources and not on the error sensors. With this modification of the cost function, the strengths of the secondary sources of the control system are tuned to drive the pressure of the error sensors to their optimal values and not to zero. This strategy leads to four more additional conclusions. The first one is that it is no longer necessary to use a number of error microphones that is greater than the number of secondary sources to ensure not only a smooth attenuation but the optimal attenuation. Second, the optimization of the control system for an enclosed sound field is limited to the optimization of the sources. Once the optimally attenuated sound field is known, there is no need to optimize the location of the error microphones. It is a matter of selecting a number of locations equal to the number of secondary sources and set the right cost function for the places selected. Third, the error transducers can be located in any place of the enclosure (i.e., far from passengers). And, lastly, this cost function can be easily rearranged in order to make the active system control a local region instead of the whole cavity.

The limitation of the cost function is that the sound field must be known, and this reduces its applicability to stationary primary sources in order to reduce the quantity of information to narrowband noises, as is the case of turboprop cabin noise. An example of the application of the proposed cost function is given using a FEM/BEM of a real turboprop. In that example, the locations of secondary sources and error sensors are found, as well as the values of residual pressure that must be achieved in each error sensor after cancellation in order to guarantee the optimal attenuation.

\section{Acknowledgment}

The authors wish to acknowledge the help and collaboration provided by Airbus Military.

\section{References}

[1] Gardonio, P., "Review of Active Techniques for Aerospace VibroAcoustic Control," Journal of Aircraft, Vol. 39, No. 2, 2002, pp. 206 214.

doi:10.2514/2.2934

[2] Baek, K. H., and Elliott, S. J., "Natural Algorithms for Choosing Source Locations in Active Control Systems," Journal of Sound and Vibration, Vol. 186, No. 2, 1995, pp. 245-267 doi:10.1006/jsvi.1995.0447

[3] Monaco, E., Franco, F., Iadevaia, M., and Lecce, L., "Interior Active Noise Control in Turbofan Aircraft: Numerical Simulation and Experimental Validation for Optimal Actuators Positioning," International Congress on Acoustics, Madrid, 2007.

[4] Bai, M., and Chang, S., "Active Noise Control of Enclosed Harmonic Fields by Using BEM-Based Optimization Techniques," Applied Acoustics, Vol. 48, 1996, pp. 15-32. doi:10.1016/0003-682X(95)00040-G

[5] Grewal, A., Zimcik, D. G., and Leigh, B., "Feedforward Piezoelectric Structural Control: An Application to Aircraft Cabin Noise Reduction,'” Journal of Aircraft, Vol. 38, No. 1, 2001, pp. 164-173. doi: $10.2514 / 2.2748$

[6] Snyder, S. D., and Hansen, C. H., "The Design of Systems to Control Actively Periodic Sound Transmission Into Enclosed Spaces, Part I: Analytical Models," Journal of Sound and Vibration, Vol. 170, No. 4, 1994, pp. 433-449.

[7] Sjösten, P., Johansson, S., Persson, P., and Claesson, I., "Considerations on Large Applications of Active Noise Control. Part II: Experimental Results," Acta Acustica United with Acustica, Vol. 89, 2003, pp. 834 843.

[8] Lau, S. K., and Tang, S. K., "Sound Fields in a Rectangular Enclosure Under Active Sound Transmission Control," Journal of the Acoustical Society of America, Vol. 110, 2001, pp. 925-938. doi:10.1121/1.1387095

[9] Palacios, I., Romeu, J., and Balastegui, A., "Two Step Optimization of Transducer Locations in Single Input Single Output Tonal Global Active Noise Control in Enclosures," Journal of Vibration and Acoustics, Vol. 132, 2010, Paper 061011. doi:10.1115/1.4002122

[10] Monson, B. B., Gee, K. L., and Sommerfeldt, S. D., "Improving Compactness for Active Noise Control of a Small Axial Cooling Fan," Noise Control Engineering Journal, Vol. 55, No. 4, 2007, pp. 397-407. doi: $10.3397 / 1.2762207$

[11] Martin, V., and Gronier, C., "Sensor Configuration Efficiency and Robustness Against Spatial Error in the Primary Field for Active Sound Control," Journal of Sound and Vibration, Vol. 264, 2001, pp. 679-704.

[12] Hinchliffe, R., Scott, I., Purver, M., and Stothers, I., "Tonal Active Control in Production on a Large Turbo-Prop Aircraft," Active 2002, Inst. of Noise ControlEngineering, Southampton, England, U.K., 2002.

[13] Emborg, U., "Cabin Noise Control in the Saab 2000 High-Speed Turboprop Aircraft," International Conference on Noise and Vibration Engineering, Univ. Of Leuven, Leuven, Belgium, 1998, pp. 13-25

[14] Grewal, A., Nitzsche, F., and Zimick, D. G., "Active Control of Aircraft Cabin Noise Using Collocated Structural Actuators and Sensors," Journal of Aircraft, Vol. 35, No. 2, 1998, pp. 324-331. doi: $10.2514 / 2.2303$

[15] Elliot, S. J., Nelson, P. A., Stothers, I. M., and Boucher, C. C., "In-Flight Experiments on the Active Control of Propeller-Induced Cabin Noise," Journal of Sound and Vibration, Vol. 140, 1990, pp. 219-238. doi:10.1016/0022-460X(90)90525-5

[16] Dorling, C. M., Eatwell, G. P., Hutchins, S. M., Ross, C. F., and Sutcliffe, S. G. C., "A Demonstration of Active Noise Reduction in an Aircraft Cabin," Journal of Sound and Vibration, Vol. 128, 1989, pp. 358-360. doi:10.1016/0022-460X(89)90779-7

[17] Palumbo, D., Cabell, R., Sullivan, B., and Cline, J., "Flight Test of Active Structural Acoustic Noise Control System," Journal of Aircraft, Vol. 38, No. 2, 2001, pp. 277-284. doi: $10.2514 / 2.2760$

[18] Johansson, S., Sjösten, P., Nordebo, S., and Claesson, I., "Comparison of Multiple- and Single. Reference MIMO Active Noie Control Approaches Using Data Measured in a Dornier 328 Aircraft," International Journal of Acoustics and Vibration, Vol. 5, No. 2, 2000, pp. 1-12.

[19] Ross, C. F., "Active Noise Control in Aircraft," 6th International Congress on Sound and Vibration, 1999, pp. 1653-1658. 
[20] Simpson, M. A., Luong, T. M., Fuller, C. R., and Jones, J. D., "FullScale Demonstration Tests of Cabin Noise Reduction Using Active Vibration Control," Journal of Aircraft, Vol. 28, No. 3, 1991, pp. 208 215 . doi: $10.2514 / 3.46014$

[21] Nelson, P. A., and Elliot, S. J., Active Control of Sound, Academic Press, San Diego, CA, 1999, pp. 327-340.

[22] Nelson, P. A., Curtis, A. R., Elliott, S. J., and Bullmore, A. J., "The Minimum Power Output of Free Field Point Sources and the Active Control of Sound," Journal of Sound and Vibration, Vol. 116, 1987, pp. 397-414. doi:10.1016/S0022-460X(87)81373-1

[23] Shafer, B. M., Gee, K. L., and Sommerfeldt, S. D., "Verification of a Near-Field Error Sensor Placement Method in Active Control of Compact Noise Sources," Journal of the Acoustical Society of America, Vol. 127, 2010, pp. EL66-EL72. doi: $10.1121 / 1.3272632$

[24] Li, D., and Hodgson, M., "Optimal Active Noise Control in Large Rooms Using a "Locally Global" Control Strategy," Journal of the Acoustical Society of America, Vol. 118, 2002, pp. 3653-3661.

[25] Elliot, S. J., Signal Processing for Active Control, Academic Press, San Diego, CA, 2001, pp. 104, 119

[26] Eriksson, L. J., "Noise and Vibration Control Engineering," Noise and Vibration Control Engineering, edited by Beraneck, L. L., and Ver, I. L., Wiley, New York, 1992, pp. 565-584.
[27] Warner, J. V., and Bernhard, R. J., "Digital Control of Local Sound Fields in an Aircraft Passenger Compartment," AIAA Journal, Vol. 28, No. 2, 1990, pp. 284-289. doi: $10.2514 / 3.10386$

[28] Ahlquist, J. R., Huguenet, P., and Palacios Higueras, J. I., "Coupled FEM/BEM Vibroacoustic Modeling of Turbopropeller Cabin Noise," 16th AIAA/CEAS Aeroacoustics Conference, AIAA Paper 2010-3948, 2010.

[29] Oslé, E., de la Gándara, J. L., and González-Díaz, A., "C295 Aircraft Global Finite Element Model: A multidisciplinary Tool Used in CASA to Reduce Time and Costs in Aircraft Certification Process," MSC 1st South European Technological Meeting, 2000.

[30] Montgomery, J. M., "Modelling of Aircraft Structural-Acoustic Response to Complex Sources Using Coupled FEM-BEM Analyses," 10th AIAA/ CEAS Aeroacoustics Conference, AIAA Paper 2004-2822, 2004.

[31] Schroeder, M. R., "The "Schroeder frequency" Revisited," Journal of the Acoustical Society of America, Vol. 99, No. 5, 1996, pp. 3240-3241. doi:10.1121/1.414868

[32] Kanev, N. G., "Effect of Errors on the Efficiency of Local Active Sound Absorbers," Acoustical Physics, Vol. 52, No. 5, 2006, pp. 571-575. doi:10.1134/S1063771006050101

[33] Baek, K. H., and Elliott, S. J., "The Effects of Plant and Disturbance Uncertainties in Active Control Systems on the Placement of Transducers," Journal of Sound and Vibration, Vol. 230, 2000, pp. 261-289. doi:10.1006/jsvi.1999.2615 


\section{Queries}

1. AU: Please check that the copyright (@) type is correct. Please note that the code will be added upon publication.

2. AU: Please provide the location of Hewlett Packard for Palacios's affiliation footnote.

3. AU: Major syntax adjustments were made throughout the paper for clarity; please read closely to confirm that your meaning was retained.

4. AU: Definitions of acronyms have been moved from the abstract into the text of the article, per AIAA style.

5. AU: "and $11 \mathrm{~dB}$ around the head of a seated crew member if the control system is tuned to achieve local control" is unclear/ incomplete. Please review and edit as necessary.

6. AU: The text reads "Shafer et al. [10] verified," but Ref. [10] is by Monson et al. Please review and edit as necessary.

7. AU: As AIAA only displays vectors in bold, please check that all variables are displayed correctly.

8. AU: Please define BPFx1 and BPFx2.

9. AU: Please define "cont." and "SHP " in the tables.

10. AU: Header rows have been added to some of the tables, per journal guidelines. Please confirm your meaning was retained.

11. AU: Instructions from AIAA indicate that figures are to appear in color only online. Please verify the usage of color in your proof is correct, and note that all figures will be grayscale in the print journal.

12. AU: If Refs. $[3,12,29]$ are published proceedings, please provide the names and locations of the publishers (NOT of the conference hosts) and the page ranges. If they are conference papers, please provide the paper numbers and the organizers' names. If they are CD-ROMs, please provide the names and locations of the CD-ROM producers.

13. AU: Please provide the issue numbers and/or months of publication for Refs. [7-9, 11, 15, 16, 22-24, 33].

14. AU: For Ref. [19], please provide the name and location of the publisher (NOT the conference host). 